\title{
LEVANTAMENTO DAS ESPÉCIES VEGETAIS EXÓTICAS E EXPERIÊNCIAS DE MANEJO NO PARQUE ESTADUAL DE PORTO FERREIRA, SP.
}

\section{Ernesto Pedro Dickfeldt ${ }^{1}$}

\section{Bruno Zuffo Janducci ${ }^{2}$}

\section{Sonia Aparecida de Souza ${ }^{3}$}

RESUMO: O programa de manejo do meio ambiente do Parque Estadual de Porto Ferreira prevê a realização de estudos e o manejo de espécies exóticas invasoras visando a conservação e a recuperação das condições primitivas da unidade. O presente trabalho apresenta o levantamento das espécies vegetais exóticas, realizado entre os anos de 2006 a 2013 nas diferentes zonas do Parque e algumas ações de manejo visando a minimização dos impactos dessas à conservação da biodiversidade. Ao todo foram identificadas 98 espécies distribuídas em 45 famílias e 89 gêneros. As famílias com maior número de espécies foram Arecaceae (9), Fabaceae (13), Poaceae (6), Rutaceae (04) e Euphorbiaceae (4). A Zona de Recuperação de Taquara e a Zona de Recuperação de Pasto são as mais infestadas, motivo pelo qual, alguns procedimentos de manejo mecânico e químico foram

\footnotetext{
${ }^{1}$ Biólogo. Técnico de Pesquisa do Parque Estadual de Porto Ferreira. E-mail: ernesto.dickfeldt@terra.com 2 Engenheiro Florestal - Secretaria do Meio Ambiente e do Desenvolvimento Sustentável de Minas Gerais. E-mail: bruno.janducci@meioambiente.mg.gov.br

3 Bióloga, Pesquisadora Científica e Gestora do Parque Estadual de Porto Ferreira. E-mail: soniasouza@if.sp.gov.br
} 
adotados. Os resultados têm revelado que os diferentes procedimentos de manejo possibilitaram o início do controle das espécies exóticas, onde a regeneração natural e o enriquecimento com espécies nativas contribuíram com a recuperação de áreas alteradas estratégicas na unidade, conforme previsto no Plano de Manejo.

Palavras-chave: Contaminação biológica. Manejo. Biodiversidade

\section{INTRODUÇÃO}

As espécies exóticas são definidas como aquelas que se encontram fora de sua área de distribuição natural e as espécies exóticas invasoras as introduzidas, que se estabelecem, dominam, formam grandes populações, ameaçam habitats, ecossistemas ou outras espécies, devido à dispersão e dominância em ambientes naturais, causando a perda da biodiversidade, sendo considerada a segunda maior ameaça mundial à biodiversidade (ZILLER, 2001; PASTORE et al., 2012).

A contaminação biológica é o processo de introdução e adaptação de espécies exóticas vegetais ou animais que não fazem parte naturalmente de um determinado ecossistema, mas que se naturalizam e provocam mudanças em seu funcionamento, causando a redução da biodiversidade e mudança da paisagem original (ZILLER, 2001).

As espécies exóticas causam problemas no desenvolvimento das nativas e podem ocasionar extinções locais, regionais, descaracterização e homogeinização dos ecossistemas, alterações nos ciclos ecológicos, mudanças no regime de incêndios naturais e rebaixamento do lençol freático (ZILLER e DECHOUM, 2007).

Segundo o Sistema Nacional de Unidades de Conservação é proibido introduzir espécies não autóctones em unidades de conservação e de acordo com o art. ํㅜ dessa lei, as ameaças à biodiversidade devem ser prevenidas, controladas e eliminadas (BRASIL, 2000). 
Como medida de conservação in situ, a Convenção da Diversidade Biológica prevê o controle ou erradicação de espécies exóticas que ameacem os ecossistemas, habitats ou espécies.

O Pacto pela Restauração da Mata Atlântica prevê ações de controle de gramíneas exóticas por meio de roçadas, aplicação de herbicida e a utilização de outros métodos de controle de competidores como capina manual ou coroamento (NAVE et al., 2010).

A contaminação por espécies exóticas é uma das principais ameaças às unidades de conservação e as áreas protegidas do Estado de São Paulo (WWF, 2000).

Ações de prevenção; detecção precoce e resposta rápida; controle; erradicação e monitoramento; capacitação técnica; informação e educação pública, pesquisa e arranjos institucionais estão previstas na estratégia paulista sobre espécies exóticas invasoras (SÃO PAULO, 2009).

$\mathrm{Na}$ Gleba Pé-de-Gigante do Parque Estadual de Vassununga a invasão por gramíneas africanas é um de seus principais impactos. Técnicas mecânicas, químicas, biológicas e de arranjo espacial foram sugeridas para o controle das mesmas (PIVELLO e KORMAN, 2005).

Estudos sobre a vegetação efetuados durante a elaboração dos Planos de Manejo, utilizando a Avaliação Ecológica Rápida revelaram a presença de 17 espécies exóticas no Parque Estadual de Vassununga e 12 na Estação Ecológica de Ribeirão Preto (SÃO PAULO, 2009b; IVANAUSKAS et al., 2012).

$\mathrm{Na}$ Reserva Biológica do Alto da Serra de Paranapiacaba os levantamentos identificaram a presença de 19 espécies exóticas invasoras e 131 espécies exóticas ruderais (PASTORE et. al., 2012).

O Parque Estadual de Porto - PEPF é também cenário de contaminação biológica por espécies vegetais exóticas, e estudos sobre essas espécies, sugestão de técnicas de manejo visando à recuperação de áreas alteradas e a substituição por nativas estão previstas no seu Plano de Manejo (TABANEZ et al., 2003). 
Assim, o diagnóstico preliminar da contaminação biológica da vegetação foi realizado no PEPF, apresentando o número de 57 espécies vegetais exóticas (JANDUCCI, 2006).

A maioria dos trabalhos sobre contaminação biológica nas unidades de conservação e áreas protegidas do Estado de São Paulo se referem aos diagnósticos e às recomendações de manejo.

Nesse trabalho apresentamos o levantamento das espécies exóticas vegetais e apresentamos algumas ações adotadas para o controle e a erradicação das mesmas em áreas estratégicas, visando à regeneração natural e a recuperação de áreas alteradas como contribuição à conservação da biodiversidade local.

\section{MATERIAIS E MÉTODOS}

O Parque Estadual de Porto Ferreira, com área de 611,55 hectares, localiza-se no município de Porto Ferreira - SP, entre as coordenadas UTM 7.579.500 e 7.583 .500 e 245.000 e 251.000 ( $21^{\circ} 49^{\prime} \mathrm{S}$ e $\left.47^{\circ} 25^{\prime} \mathrm{W}\right)$.

Segundo a classificação de Köppen, o clima é do tipo Cwa, mesotérmico de inverno seco, com temperaturas inferiores a $18^{\circ} \mathrm{C}$ no inverno e superiores a $22^{\circ} \mathrm{C}$ no verão. O relevo possui declividade entre 0 e $5 \%$ (plano a suave ondulado) e quotas altimétricas entre 540 e 560 metros (TABANEZ et al., 2003).

O levantamento das espécies exóticas ocorreu entre os anos de 2006 à junho de 2013, utilizando as trilhas e o leito do ribeirão dos Patos durante as atividades de fiscalização, monitoramento ambiental e pesquisas nas diferentes zonas de manejo do Parque. O maior esforço de identificação ocorreu na vegetação de borda.

$\mathrm{Na}$ zona de uso especial estão localizados os próprios estaduais utilizados como escritórios, galpão operacional, alojamento, guarita e residências.

$\mathrm{Na}$ zona de uso intensivo encontram-se o estacionamento, o Centro de Visitantes, os quiosques, o Playgrond, o Recanto da Harmonia e o Arboreto Orlando Prezotto. 
As trilhas, os caminhos e as estradas têm seus percursos na Zona de Uso Extensivo.

A Zona de Recuperação se refere à áreas com presença de gramíneas e outras espécies exóticas.

A Figura 1 apresenta um croqui do mapa do zoneamento do PEPF.

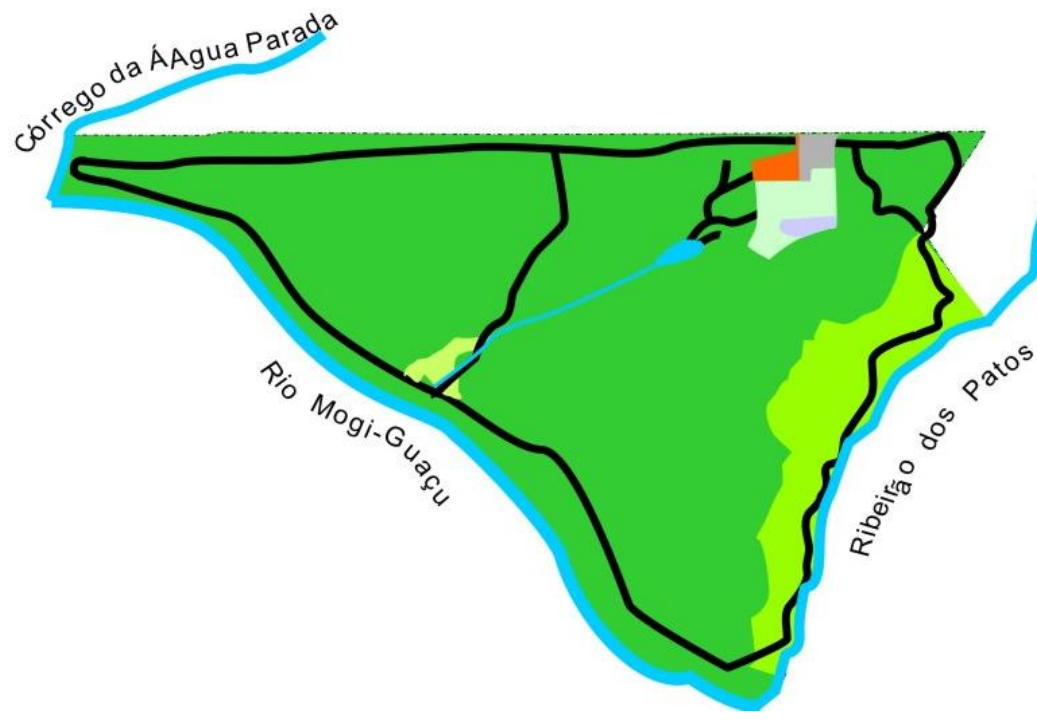

Zona Primitiva

Zona de Uso Extensivo

Zona de Uso Especial

Zona de Uso Intensivo

Zona de Recuperação de Taquara

Zona de Recuperação de Pasto

Zona de Recuperação de Jequitibás

Para a identificação das espécies vegetais exóticas foram utilizadas as referências de Lorenzi (2000); Ziller (2000) Ziller e Rosa (2001); Camargo et al.(2006); Janducci (2006); Pastore et al. (2012). Os indivíduos foram marcados e após a classificação da espécie foi definindo o seu grau de disseminação em pouco disseminada, moderadamente disseminada e amplamente disseminada. 
As espécies não identificadas no campo foram coletadas, quando possível com presença de material fértil e identificadas através da coleção do Herbário "Irina Delanova De Gemtchujnicov" - Herbário BOTU, da UNESP de Botucatu.

\section{RESULTADOS E DISCUSSÃO}

No PEPF foram identificadas 98 espécies exóticas vegetais de 89 gêneros pertencentes a 45 famílias botânicas, considerando as formações vegetais nativas de cerrado e floresta estacional semidecidual do Estado de São Paulo.

A Tabela 1 apresenta a relação das espécies, a localização e o grau de disseminação que se encontram.

Tabela 1. Espécies exóticas do Parque Estadual de Porto Ferreira. As espécies invasoras estão destacadas com *. ZUE= Zona de Uso Especial, ZEX= Zona de Uso Extensivo, ZUl= Zona de Uso Intensivo, $\mathrm{ZR}=$ Zona de Recuperação. $\mathrm{PD}=$ Pouco Disseminada, $\mathrm{MD}=$ Moderadamente Disseminada e $\mathrm{AM}=$ Amplamente Disseminada.

\begin{tabular}{|c|c|c|c|}
\hline Família/Espécie & Nome popular & Localização & Disseminação \\
\hline \multicolumn{4}{|l|}{ Agavaceae } \\
\hline Agave attenuata & agave & ZUE & PD \\
\hline \multicolumn{4}{|l|}{ Anacardiaceae } \\
\hline Mangifera indica & mangueira & ZUE & PD \\
\hline Spondias lutea & cajá & ZUI & PD \\
\hline Spondias purpúrea & seriguela & ZUE & PD \\
\hline \multicolumn{4}{|l|}{ Annonaceae } \\
\hline Anona squamosa & biribá & ZUE & PD \\
\hline Anona muricata & gravioleira & ZUI & PD \\
\hline \multicolumn{4}{|l|}{ Apocynaceae } \\
\hline Allamanda puberula & alamanda & ZUE & PD \\
\hline \multicolumn{4}{|l|}{ Araliaceae } \\
\hline \multirow{2}{*}{\multicolumn{4}{|c|}{ Araceae }} \\
\hline & & & \\
\hline Caladium sp & caládio & ZUE & PD \\
\hline \multicolumn{4}{|l|}{ Araucariaceae } \\
\hline Araucaria angustifolia & Pinheiro-do-paraná & ZUE & PD \\
\hline
\end{tabular}


Areca triandra

Attalea attaleiodes

Attalea dahlgreniana

Attalea sp.

Caryota urens

Dypsis madascariensis

Phoenix roebelenii

Raphis excelsa

Sabal marítima

Bignoniaceae

Jacaranda mimosifolia

Spathodea campanulata

Tabebuia pentaphylla

Bixaceae

Bixa orellana

\author{
areca \\ palha-branca \\ perinão \\ atalea \\ palmeira-rabo-de-peixe \\ areca-de-Cuba \\ tamareira-de-jardim \\ ráfis \\ sabal-de-Cuba
}

jacarandá-mimoso

espatódea

Ipê-de-el-salvador

urucum

$\begin{array}{ll}\text { ZUE } & P D \\ \text { ZUE } & P D \\ \text { ZUE } & P D \\ \text { ZUE } & P D \\ \text { ZUE } & P D \\ \text { ZUE } & P D \\ \text { ZUI } & P D \\ \text { ZUI } & P D \\ \text { ZUE } & P D\end{array}$

ZUI PD

ZUE $\quad P D$

ZUI PD

continua...

continuação da Tabela 1.

\begin{tabular}{|c|c|c|c|}
\hline Família/Espécie & Nome popular & Localização & Disseminação \\
\hline \multicolumn{4}{|l|}{ Cactaceae } \\
\hline Opuntia cochenillifera & cactus & ZR & PD \\
\hline \multicolumn{4}{|l|}{ Caricaeae } \\
\hline Carica papaya & mamoeiro & ZUE & PD \\
\hline \multicolumn{4}{|l|}{ Combretaceae } \\
\hline Terminalia sp. & fruto-de-veado & ZUE & PD \\
\hline \multicolumn{4}{|l|}{ Comelinaceae } \\
\hline Tradescantia zebrina & lambari & ZUE & PD \\
\hline \multicolumn{4}{|l|}{ Cycadaceae } \\
\hline Cycas circinalis & cica & ZUE & PD \\
\hline \multicolumn{4}{|l|}{ Ericaceae } \\
\hline \multicolumn{4}{|l|}{ Euphorbiaceae } \\
\hline Codiaeum variegatum & cróton & ZUE & PD \\
\hline Euphorbia cotinifolia & euforbia & ZUE & PD \\
\hline Euphorbia pulcherrina & bico-de-papagaio & ZUE & PD \\
\hline Manihot utilíssima & mandioqueira & ZUI & PD \\
\hline \multicolumn{4}{|l|}{ Fabaceae } \\
\hline Albizia lebbeck & coração-de-negro & ZUI & PD \\
\hline Amburana cearensis & amburana & ZUE & PD \\
\hline Anadenanthera pavonina & olho-de-pavão & ZUI & PD \\
\hline
\end{tabular}


Bauhinia variegata

Cassia grandis

Cassia fistula

Caesalpinia echinata

Caesalpinia ferrea v. leiostachya

Caesalpinia peltophoroides

Clitoria fairchildiana

Dalbergia nigra

Leucaena leucocephala

Schizolobium parahiba

Iridaceae

Dietes bicolor

Lamiaceae

Plectranthus barbatus

Lauraceae

Cinnamomum zeylanicum

Ocotea porosa

Persea americana

Lecythidaceae

Gustavia augusta

Lecythis pisonis

Lythraceae

Cuphea gracilis

Lagerstroemia indica

Magnoliaceae

Michellia sp.

Malpighiaceae

Lophantera lactescens

Malpighia glabra

Malvaceae

Apeiba tibourbou

\author{
pata-de-vaca \\ cássia-grande \\ cássia-imperial \\ pau-brasil \\ pau-ferro \\ sibipiruna \\ sombreiro
}

jacarandá-da-Bahia

leucena

guapuruvu

moréia

boldo

canela

imbuia

abacateiro

jeniparana

sapucaia

erica

magnólia

lofantera-da-amazônia

aceroleira

escova-de-macaco
ZUE

ZUI

ZUE

ZUE

ZUE

ZUE

ZUE

ZUE

ZUE

ZUI

ZUE

ZUE

ZUI

ZUE

PD

PD

ZUE $\quad P D$

ZUI PD

ZUE $\quad P D$

ZUE PD

ZUE PD

ZUI PD

ZUE PD

ZUI PD

continuação da Tabela 1.

\begin{tabular}{lccc}
\hline \multicolumn{1}{c}{ Família/Espécie } & Nome popular & Localização & Disseminação \\
\hline Hibiscus sp. & hibísco & ZUE & PD \\
Pachira aquatica & monguba & ZUI ZUE & PD \\
$\begin{array}{l}\text { Sterculia striata } \\
\text { Meliaceae }\end{array}$ & chichá & ZUI & PD \\
Azadirachta indica & nim & ZUI & PD \\
$\begin{array}{l}\text { Swietenia macrophylla } \\
\text { Moraceae }\end{array}$ & mogno & ZUI & PD \\
Artocarpus integrifolius & & & \\
Morus nigra & jaqueira & ZUE & PD \\
& amoreira & ZUE & PD
\end{tabular}




\begin{tabular}{|c|c|c|c|}
\hline \multicolumn{4}{|l|}{ Musaceae } \\
\hline Musa sp. & bananeira & ZUE & PD \\
\hline Campomanesia phaea & cambuci & ZUI & $\mathrm{PD}$ \\
\hline Eucapiptus sp. & eucalipto & ZEX & PD \\
\hline Psidium guajava & goiabeira & ZUE & PD \\
\hline Syzigium cumini & jambolão & ZUE,ZUI,ZR & MD \\
\hline \multicolumn{4}{|l|}{ Nyctaginaceae } \\
\hline Bougainvillea sp. & primavera & ZUE & PD \\
\hline \multicolumn{4}{|l|}{ Oleaceae } \\
\hline Ligustrum sinense & variegata & ZUE & PD \\
\hline \multicolumn{4}{|l|}{ Orchidaceae } \\
\hline Dendrobium nobile & dendróbium & ZUE & PD \\
\hline Zeuxine strateumatica & orquídea & ZUI & PD \\
\hline \multicolumn{4}{|l|}{ Pinaceae } \\
\hline Pinus sp. * & pinus & ZR & $A D$ \\
\hline \multicolumn{4}{|l|}{ Phytolaccaceae } \\
\hline Petiveria alliacea $\mathrm{L}$. & guiné & ZP & PD \\
\hline \multicolumn{4}{|l|}{ Poaceae } \\
\hline Brachiaria decubens* & braquiaria & ZUE,ZEX,ZUI,ZR & $A D$ \\
\hline Melinis minutiflora & capim-gordura & ZR & MD \\
\hline Panicum maximum & capim-colonião & ZUE,ZEX,ZR & MD \\
\hline Pennisetum pupureum & napiê & ZR & PD \\
\hline Phyllostachys sp* & vara-de-pescar & ZR & $A D$ \\
\hline Zoysia japônica & grama-esmeralda & ZUE,ZUI & MD \\
\hline \multicolumn{4}{|l|}{ Proteaceae } \\
\hline Grevillea robusta & grevilha & ZUE & PD \\
\hline \multicolumn{4}{|l|}{ Rosaceae } \\
\hline Eriobotrya japônica & nêspera & ZUE & PD \\
\hline Rosa chinensis & rosa & ZUE & PD \\
\hline \multicolumn{4}{|l|}{ Rubiaceae } \\
\hline Coffea arábica & café & ZP & PD \\
\hline \multicolumn{4}{|l|}{ Ruscaceae } \\
\hline Sansevieira trifasciata & espada-de-São-Jorge & ZP,ZUE & MD \\
\hline \multicolumn{4}{|l|}{ Rutaceae } \\
\hline Citrus sp 1. & laranjeira & ZUE & PD \\
\hline Citrus sp 2. & limoeiro & ZUE,ZP & PD \\
\hline Clausena sp. & clausena & ZUE & PD \\
\hline Murraya paniculata & murta & ZUE & PD \\
\hline \multicolumn{4}{|l|}{ Schophulariaceae } \\
\hline Veronica próstata & miosotis & ZUE & PD \\
\hline
\end{tabular}


continuação da Tabela 1.

\begin{tabular}{|c|c|c|c|}
\hline Família/Espécie & Nome popular & Localização & Disseminação \\
\hline \multicolumn{4}{|l|}{ Solanaceae } \\
\hline Brunfelsia uniflora & manacá-de-cheiro & ZUE & PD \\
\hline Capsicum baccatum & pimenta-vermelha & ZUE & PD \\
\hline \multicolumn{4}{|l|}{ Strelitziacaceae } \\
\hline Ravenala madagasscariensis & árvore-do-viajante & ZUE & PD \\
\hline \multicolumn{4}{|l|}{ Verbenaceae } \\
\hline Duranta repens aurea & pingo-de-ouro & ZUE & PD \\
\hline \multicolumn{4}{|l|}{ Zingiberaceae } \\
\hline Hedychium coronaruim & lírio-do-brejo & ZP & PD \\
\hline
\end{tabular}

As famílias com maior número de espécies no PEPF foram Fabaceae (13), Arecaceae (9), Poaceae (6), Rutaceae (4) e Euphorbiaceae (4). No Parque Estadual de Vassununga as mais representativas foram Poacea (7) e Mirtaceae (3), enquanto que na Estação Ecológica de Ribeirão Preto Fabaceae (3) e Meliaceae (3).

As espécies Pinus sp. Brachiaria decumbens e Phyllostachys sp atualmente apresentam comportamento invasivo no PEPF.

Inferimos que o número de espécies exóticas (98) no PEPF está relacionado ao fato da área ter pertencido à uma Fazenda antes de sua criação, onde diversas espécies frutíferas, ornamentais e medicinais foram plantadas ao redor das moradias, bem como para a formação de cortina vegetal entre as casas e a rodovia. Outro fator é o esforço de sete anos de levantamento.

A maioria das espécies encontra-se na Zona de Uso Especial, Zona de Uso Intensivo e Zona de Recuperação.

A espécie Brachiaria decubens ocorreu em todas as zonas do Parque, apresentando-se como a mais invasiva.

As espécies frutíferas Anona squamosa, Anona muricata, Eryobotrya japônica, Mangifera indica, Spondia purpurea, Carica papaya, Persea americana, Malpighia glabra, Artocarpus integrifolius, Morus nigra, Psidium guajava, Musa sp., Citrus sp1 e Citrus sp2, 
encontram-se pouco disseminadas e a maioria localiza-se na Zona de Uso Especial e de Recuperação. O monitoramento realizado nos últimos anos indicou que as mesmas não apresentam problemas de contaminação em relação à Zona Primitiva ou de Recuperação.

As espécies Agave attenuata, Allamanda puberula,Scheefflera actinophylla, Calladium sp, Areca trianda, Attalea attalenoides, Attalea dahlgreniana, Attalea sp., Caryota urens, Dypsis madascariensis, Raphis excelsa, Sabal marítima, Spathodea campanulata, Opuntia cochenillifera, Tradescantia zebrina, Cycas circinalis, Rhododendron simsii, Codieaum variegatum, Euphorbia cotinifolia, Euphorbia pulcherrima, Cassia fistula, Dietes bicolor, Cuphea gracilis, Lagerstroemia indica, Michelia sp, Hibiscus sp. Bouganvillea sp., Ligustrum sinense, Dendrobium nobile, Zoysia japônica, Grevillea robusta, Rosa chinensis, Murraya paniculata, Sansevieiria trifasciata, Veronica prostata, Brunfelsia uniflora, Ravenala madagascariensis, Duranta repens aurea foram utilizadas na ornamentação e na jardinagem na Zona de Uso Especial e na Zona de Uso Intensivo. Essas espécies também não apresentaram potencial de disseminação em relação à vegetação natural da unidade.

Coffea arabica, Pettiiveria alliacea L. Hedycium coronarium são algumas das espécies exóticas pouco disseminadas, encontrados na Zona Primitiva da unidade, que em breve devem ser erradicadas.

No estacionamento foram plantadas mudas de pau-brasil com fins educativos para abordagem da história de devastação das florestas. Acredita-se que essa espécie não apresente problemas de contaminação, sendo necessário o seu monitoramento.

No Arboreto Orlando Prezotto encontram-se as espécies Spondias lutea, Jacaranda mimosifolia, Anadenanthera pavonina, Cassia grandis, Caesalpinia echinata, Ocotea porosa, Lophantera lactescens, Apeiba tibourbou, Pachira aquatica, Swietenia macrophylla, Campomanesia phaea,Terminalia sp., Clitoria fairchildiana, Shizolobium parahiba, Lecythis pisonis, Sterculia striata consideradas exóticas na região e Azadirachta indica e Annona muricata exótica no Brasil foram trazidas por funcionários antigos e por membros da comunidade local que procuravam áreas para plantio de mudas em datas comemorativas como a Semana do Meio Ambiente e da Árvore. 
Atualmente há uma preocupação em relação à escolha de espécies para plantio, bem como a realização de monitoramento das existentes nas diferentes zonas do Parque, tendo em vista o problema de contaminação biológica e legislação vigente.

As Figuras de 2 a 4 ilustram as áreas do PEPF infestadas por espécies vegetais exóticas.

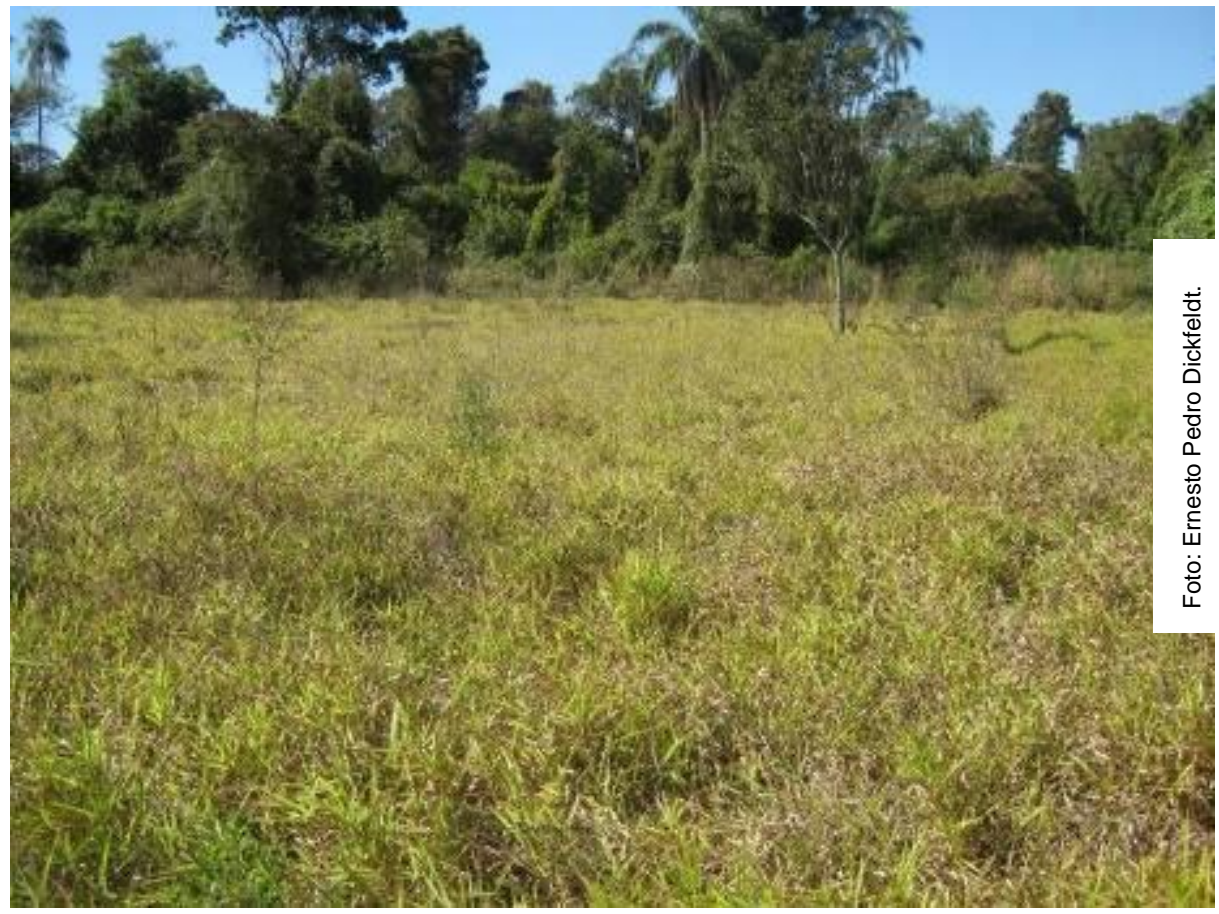

Figura 2. Zona de Recuperação de Pasto infestada por Brachiaria decumbens. 


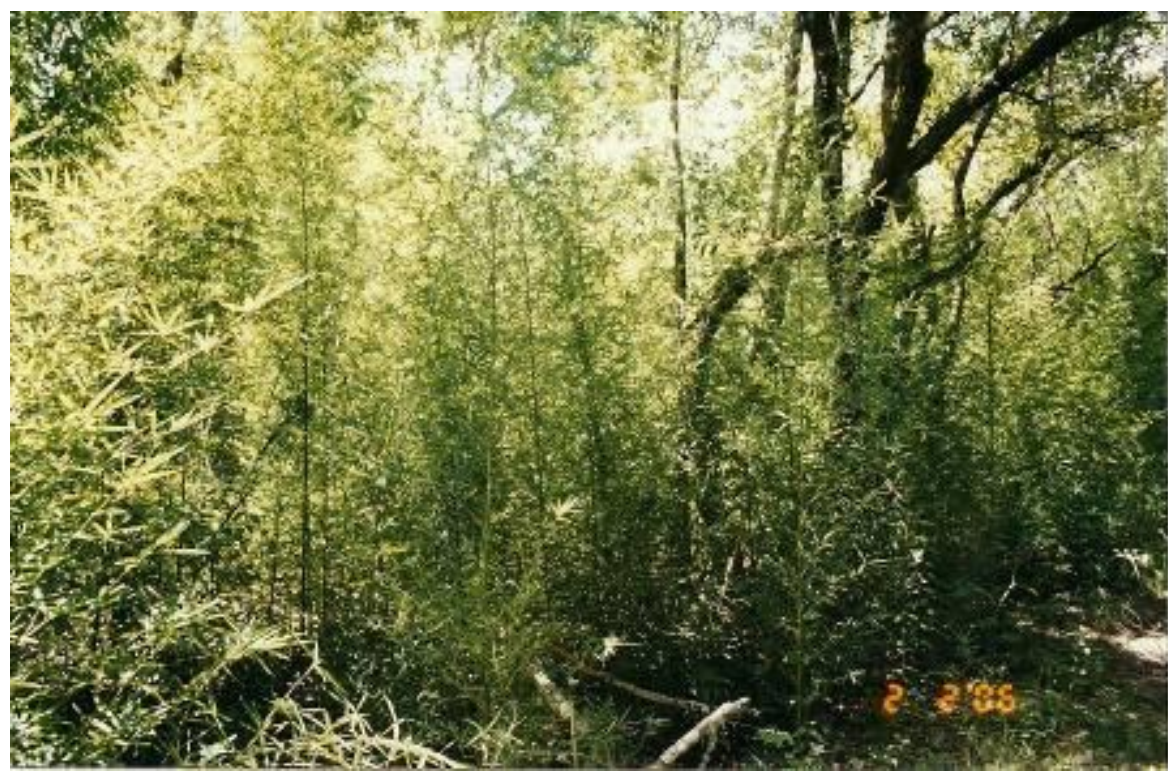

Figura 3. Área infestada com Phyllostachys sp.

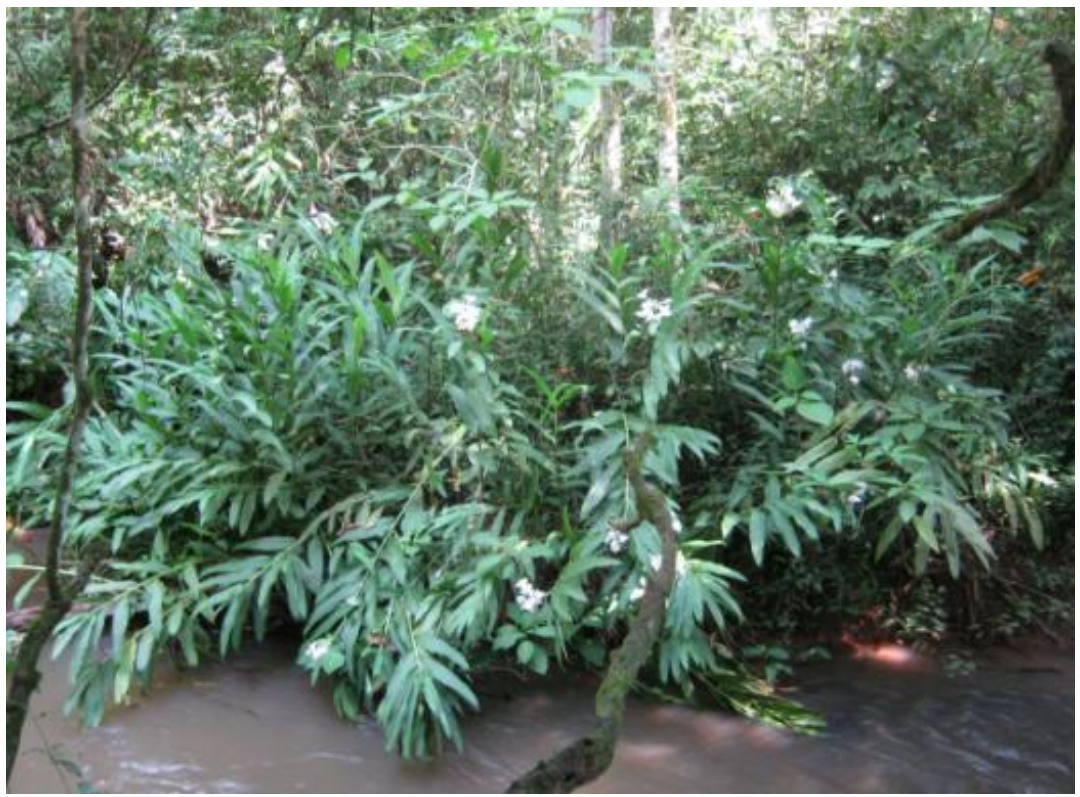

Figura 4. Moita isolada de Hedychium coronarium lírio do brejo às margem do ribeirão dos Patos, limite leste do PEPF. 
As espécies Phyllostachys sp e Brachiaria decubens por estarem amplamente disseminadas, têm sido alvo de ações de manejo na unidade.

\section{EXPERIÊNCIAS DE MANEJO}

Considerando a vulnerabilidade do Parque pelo seu tamanho, uso e ocupação do solo no entorno, a falta de conectividade com outras áreas naturais e a necessidade de conservação da biodiversidade e dos recursos naturais, algumas ações foram implantadas visando à erradicação e o controle de algumas espécies exóticas.

4.1 Manejo em área com bambu do gênero Phyllostachys sp.

Entre os anos de 2006 à 2009 uma área de $1.860 \mathrm{~m}^{2}$ na Zona de Recuperação de Taquara foi manejada adotando-se os procedimentos de corte e retirada manual de colmos; roçada com roçadeira hidráulica; sulcamento; rastelamento; enleiramento; retirada manual de rizomas remanescentes; abertura de vala no perímetro da área manejada (Figura 5), monitoramento e manutenção. A proximidade da floresta e a presença de banco de sementes favoreceram a regeneração natural, não sendo necessários esforços de enriquecimento (Dickfeldt et al., 2011).

O monitoramento da área ainda é efetuado anualmente. 


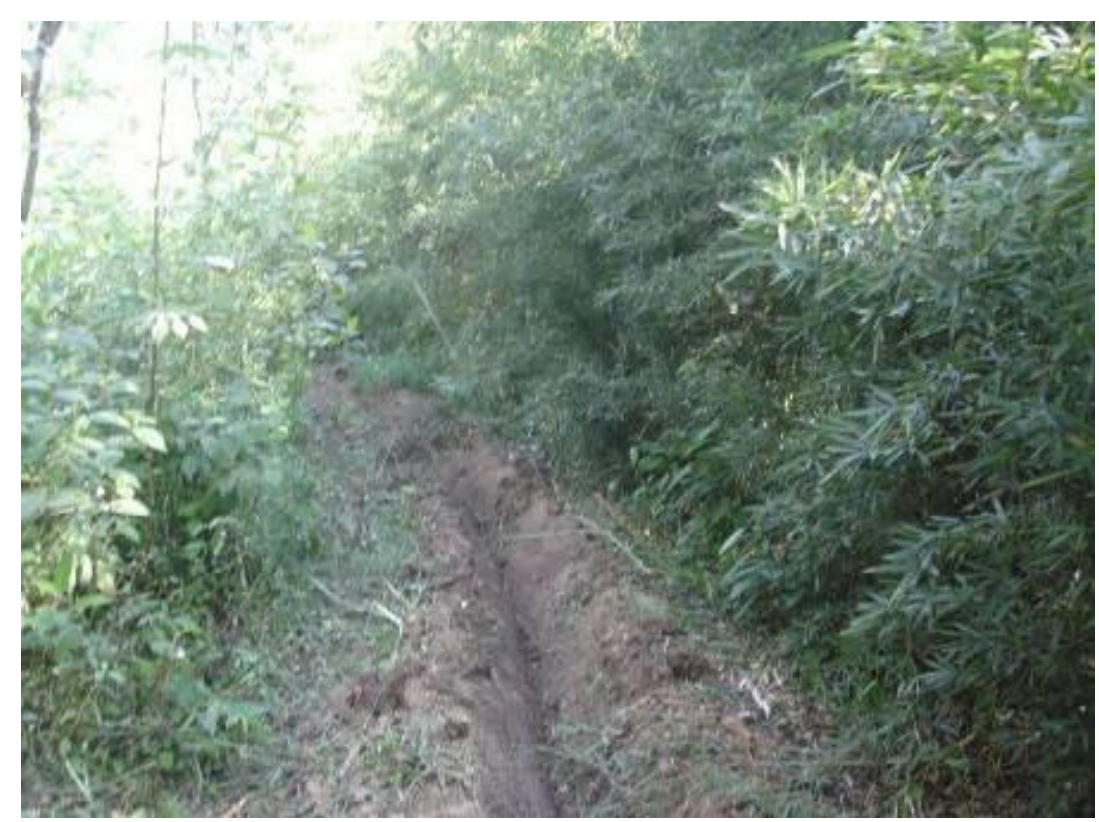

Figura 5. Vala aberta para controle do crescimento de rizomas de Phyllostachys sp.

4.2 Manejo na Zona de Recuperação de Pasto.

Nessa zona havia gado na época em que a área pertencia à Fazenda Santa Mariana. Com a criação da unidade de conservação o pasto foi desativado, porém, a presença de gramíneas dificultou a regeneração natural da vegetação.

Em atendimento às recomendações do Plano de Manejo, no ano de 2012 iniciaram as ações de manejo mecânico com trator e roçadeira hidráulica, e controle químico com aplicação de glifosato em área de 2,9 hectares (Figuras 6 e 7). 


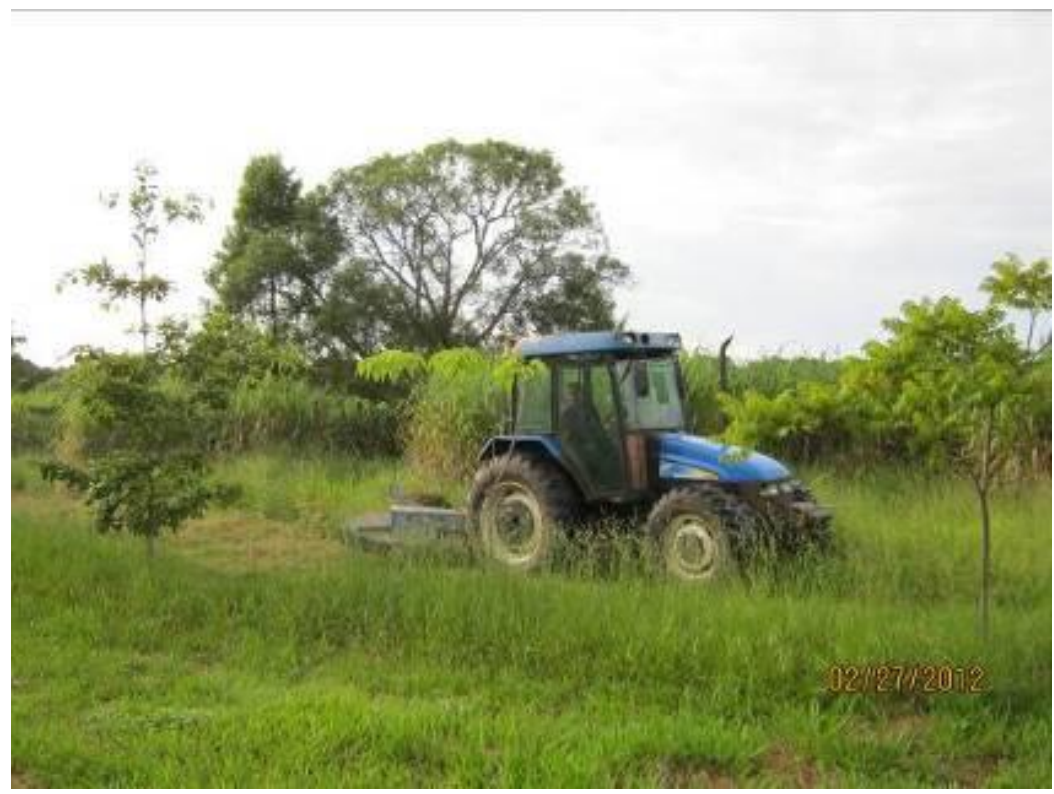

Figura 6. Roçada mecânica de Brachiaria decubens na Zona de Recuperação de Pasto do PEPF.

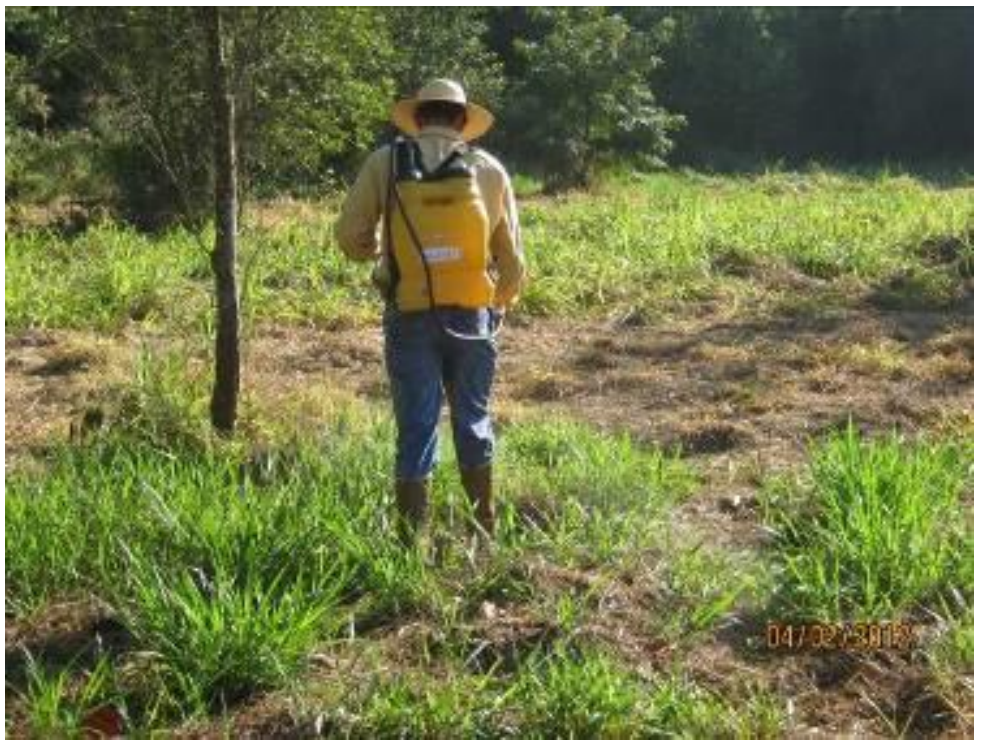

Figura 7. Controle químico da rebrota de Brachiaria decubens na Zona de Recuperação de Pasto do PEPF.

Após o manejo foi realizado o enriquecimento com o plantio de espécies nativas (Figura 8) destacando as seguintes: Acrocomia aculeata (Jack.) Lodd ex Mart.- macaúva, 
Campomanesia adamantium (Cambess.) O. Berg - gabiroba-do-cerrado, Casearia sylvestris Sw.- guaçatonga, Copaifera langsdorffii Desf. - copaíba, Dipterix alata Vogel - cumbaru, Eugenia uniflora L. - pitanga, Ficus guaranítica Schodat - figueira, Hexaclamys edulis (O.Berg) Kausel \& D. Legrand - pêssego-do-mato, Hymenaea courbaril var. stilbocarpa (Hayne) Y.T.Lee \& Langenh - jatobá, Myrciaria cauliflora (Mart.) O. Berg - jabuticabeira, Psidium guineense Sw. - aracá-do-campo, Rhamnidium elaeocarpus Reissek - saguaraji e Syagrus romanzoffiana (Cham.) Glassman - jerivá.

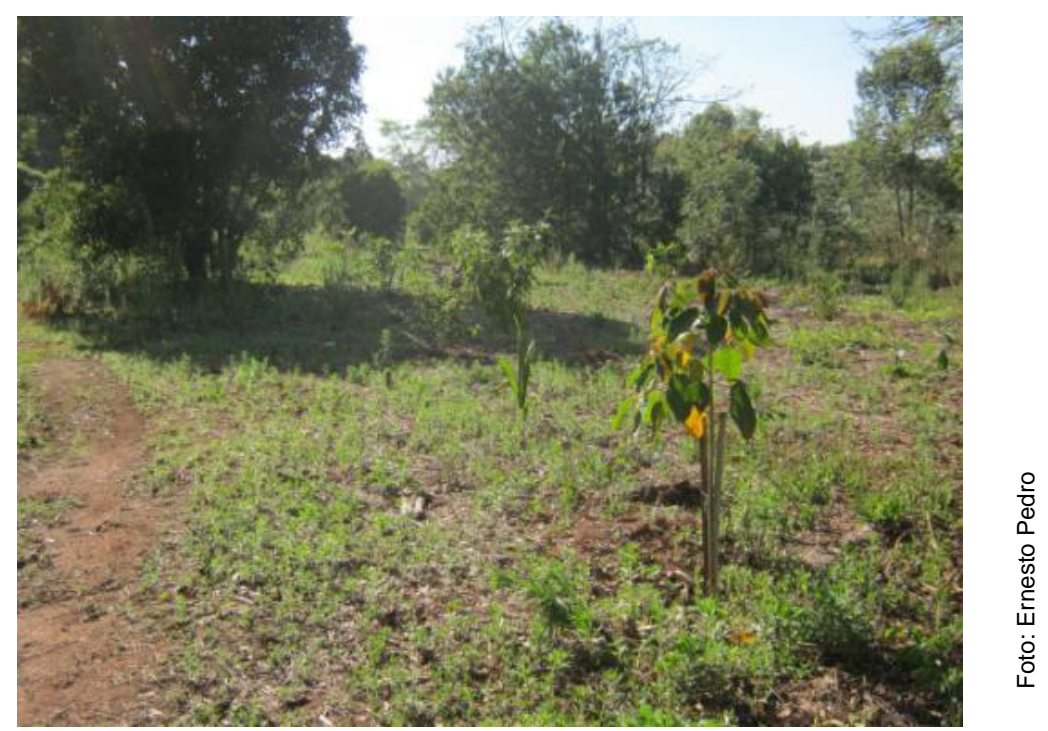

Figura 8. Vista do enriquecimento na Recuperação de Pasto com destaque para o plantio de muda de Ficus guaranitica Schodat - figueira.

A escolha da maioria das espécies teve também o objetivo de disponibilizar recursos alimentares para a fauna em médio prazo e contribuir com a redução do número de acidentes com animais silvestres que são atropelados ao saírem do Parque a procura de alimento nas propriedades vizinhas. 


\section{CONCLUSÃO}

Em atendimento ao Plano de Manejo e às políticas públicas, o diagnóstico das espécies vegetais exóticas e as ações de controle e manejo têm sido realizados visando à minimização da contaminação biológica, favorecendo a recuperação de áreas alteradas e a conservação da biodiversidade no interior do Parque Estadual de Porto Ferreira.

Esporadicamente são realizadas ações de monitoramento nas diferentes zonas do Parque para verificar a disseminação das espécies com potencial invasivo.

Estudos e investimentos são necessários para ampliar as ações de manejo visando o controle e a erradicação de espécies vegetais exóticas em outras áreas estratégicas da unidade de conservação.

\section{REFERÊNCIAS}

BRASIL. Convenção sobre Diversidade Biológica: Conferência para Adoção do Texto Acordado da CDB - Ato Final de Nairobi. Brasília: MMA/SBF, 2000. p. 14 (Biodiversidade 2).

CRESTANA, M.S.M. et al. Florestas Sistemas de Recuperação com Essências Nativas, Produção de Mudas e Legislações. 2. ${ }^{\text {a }}$ ed. Campinas, CATI, 2006. p. 62-78.

DICKFELDT, E.P. et al. Manejo de bambu exótico invasor Phyllostachys sp. no Parque Estadual de Porto Ferreira, Porto Ferreira - SP, Brasil. In: Congreso Internacional de Áreas Protegidas, 7; Convención Internacional sobre Medio Ambiente y Desarrollo, 8, La Havana - Cuba, 2011. AP 108. p.481-491. CD ROM.

IVANAUSKAS, N. M. Vegetação da Estação Ecológica de Ribeirão Preto: Caracterização e Subsídios ao Plano de Manejo. IF Sér. Reg. n. 45 p. 1-47 set. 2011. Disponível em: http://www.iflorestal.sp.gov.br/publicacoes/serie_registros/index.asp. Acesso em 05.02.2013.

JANDUCCI, B.Z. Diagnóstico preliminar da contaminação biológica do Parque Estadual de Porto Ferreira. Relatório de Estágio Curricular Supervisionado para obtenção do título de Engenheiro Florestal, Faculdade de Ciências Agronômicas, Universidade Estadual Paulista. Campus Botucatu, Botucatu-SP. 2006, 40 p.

LORENZI, H. Plantas Daninhas do Brasil. Instituto Plantarum de estudos da flora LTDA. 3 ed. São Paulo: Nova Odessa, 2000. 608 p. 
NAVE, A.G. et al.Descrição das ações operacionais de restauração. In: RODRIGUES, R.R., BRACATION, P.H.S., ISERNHAGEN (Coord.). Pacto pela restauração da Mata Atlântica: referencial dos conceitos $e$ ações de restauração florestal. LCB/ESALQ/USP. 3a․ ed. rev.- São Paulo: LERF. 2010, p.180-242.

PASTORE, M. et al. Plantas exóticas invasoras na Reserva Biológica do Alto da Serra de Paranapiacaba, Santo André - SP: guia de campo. São Paulo: Instituto de Botânica, 2012. 46 p. Disponível em www.ibot.sp.gov.br/publicacoes/virtuais/guia\%20de\%20campo.pdf. Acesso em 05.02.2013.

PIVELLO, V.R.; KORMAN, V. O desafio da conservação dos recursos naturais na região. Conservação e Manejo da biodiversidade. In: PIVELLO, V.R.; VARANDA, E.M. (Org.). 0 Cerrado Pé-de-Gigante (Parque Estadual de Vassununga): Ecologia e Conservação. São Paulo: SMA, 2005. p. 298-309.

SÃO PAULO. Resolução SMA no 033, de 22 de maio de 2009. Dispõe sobre a criação de Grupo de Trabalho para o desenvolvimento de proposta para "Estratégia Estadual sobre Espécies Exóticas Invasoras". Disponível em: www.ambiente.sp.gov.br/legislacao/estadual/resolucoes/2009_res_est_sma_33.pdf. Acesso em: 20 fev. 2010.

SÃO PAULO. Plano de Manejo do Parque Estadual de Vassununga. Santa Rita do Passa Quatro, SP. 328p. 2009. Não publicado (em análise no Conselho Estadual de Meio Ambiente - CONSEMA).

SOUZA, V.C.; LORENZI, H. Guia ilustrado para identificação das famílias de Angiospermas da flora brasileira, baseado em APG II. Nova Odessa: Instituto Plantarum, 2008. $704 \mathrm{p}$.

TABANEZ, M.F. et al. Plano de Manejo do Parque Estadual de Porto Ferreira. São Paulo: $\mathrm{O}_{2}$ Estúdio Web, 2003. 1 CD-ROM.

WWF-Brasil. Instituto Florestal do Estado de São Paulo/ Fundação Florestal do Estado de São Paulo, 2004. RAPPAM: Implementação da Avaliação Ecológica Rápida e Priorização do Manejo de Unidades de Conservação do Instituto Florestal e da Fundação Florestal. São Paulo.

ZILLER, S.R. A Estepe Gramíneo-Lenhosa no segundo planalto do Paraná: diagnóstico ambiental com enfoque à contaminação biológica. 2000. 268 f. Tese (Doutorado em Ciências Agrárias): Universidade Federal do Paraná. Curitiba. 
ZILLER, S.R; ROSA, F.L.O. Perda de Biodiversidade em Áreas Protegidas pela Invasão de Espécies Exóticas. In: SIMPÓSIO DE ÁREAS PROTEGIDAS. PESQUISA E DESENVOLVIMENTO SÓCIO-ECONÔMICO. I. Anais. 2001. Pelotas. Out.2001. Educat, 2001 p.229-233.

ZILLER, S.R.; DECHOUM, M.S. Degradação ambiental causada por plantas exóticas invasoras e soluções para o manejo em unidades de conservação de proteção integral. In: BARBOSA, L.M.; SANTOS Jr., N.A.S. (Org.). CONGRESSO NACIONAL DE BOTANICA, 58., 2007. São Paulo. Anais... São Paulo: Instituto de Botânica, 2007. p.365-360.

ZILLER, S. R. Programa de Espécies Exóticas Invasoras Para a América do Sul. The Nature Conservancy - Brasil. Modelo para o desenvolvimento de uma estratégia nacional para espécies exóticas invasoras. Disponível em: http://i3n.iabin.net/documents/pdf/Modeloestrategianacionalinvasoras-portugues pdf. Acesso em 27 jul. 2009. 